\title{
Three-Dimensional Topography Simulation for Deposition and Etching Processes Using a Level Set Method
}

\author{
A. Sheikholeslami, C. Heitzinger, T. Grasser, and S. Selberherr
}

\begin{abstract}
We present the application of level set and fast marching methods to the simulation of surface topography of a wafer in three dimensions for deposition and etching processes. These simulations rest on many techniques, including a narrow band level set method, fast marching for the Eikonal equation, extension of the speed function, transport models, visibility determination, and an iterative equation solver.
\end{abstract}

\section{INTRODUCTION}

To understand the influence of edge topography on device characteristics, which is important for highly integrated ICs, an accurate three-dimensional topography simulator is required. However, topography simulation is still faced with many challenges which limit its general applicability and usefulness. In addition, threedimensional topography simulation tends to be very CPU and memory intensive to date.

Various surface representation algorithms have been used for the development of three-dimensional topography simulators [6]. Roughly speaking, these algorithms fall into three categories.

- Segment-based models, such as the facet motion model [8], [9]: In this model a nodal triangularization of the interface is used. The position of the nodes is then updated by determining front information about the normals and curvature of surface facets. Because interstices or duplications between neighboring surface facets occur during their advance along the normal, area-readjustment procedures are needed. However, these procedures induce significant computational error into the simulation result in proportion to the complexity of the process geometry.

- Cell-based models, such as the cellular model [3], [5]: These models can easily handle topological changes and can be extended to three dimensions, whereas the determination of geometric quantities such as surface normals and curvature can be inaccurate.

A. Sheikholeslami, C. Heitzinger, and S. Selberherr are with the Institute for Microelectronics, Technical University Vienna, Gußhausstraße 27-29/E360, A-1040 Vienna, Austria, E-mail: sheikholeslami@iue.tuwien.ac.at

T. Grasser is with the Christian Doppler Laboratory for TCAD in Microelectronics at the Institute for Microelectronics, E-mail: grasser@iue.tuwien.ac.at
- Level set method-based models [1], [2], [12]: In this method the interface extraction is based on the solution of a hyperbolic partial differential equation. The location of an interface is the zero level set of a higher dimensional function called level set function. This model provides an interesting alternative method for solving the above mentioned problems.

Based on an efficient and precise level set method including narrow banding and extending the speed function in a sophisticated algorithm, we have developed a general three-dimensional topography simulator for the simulation of deposition and etching processes. The simulator is called ELSA (Enhanced Level Set Applications) and works efficiently concerning computational time and memory consumption, and it simultaneously ensures high resolution.

The outline of this paper is as follows. First, we present an optimized method to obtain the initial level set function. Second, we describe briefly how to extend the speed function combined with narrow banding using a fast marching method. Third, the stability and the complexity of the simulator is discussed. Fourth, we present the transport models. Finally, simulation results are shown.

\section{INITIALIZATION}

The basic idea of the level set method is to view the curve or surface in question at a certain time as the zero level set (with respect to the space variables) of a certain function called level set function. Each point on the surface is moved with a certain speed normal to the surface and this determines the time evolution of the surface. For points on the zero level set the speed function is usually determined by physical models and in our case by the etching and deposition processes, or more precisely by the fluxes of certain gas species and subsequent surface reactions. The speed function at grid points has to be extended from the known values of the speed function on the surface. We will discuss this more in detail in the next section.

In order to apply the level set method a suitable initial function has to be determined. A good choice is the signed distance function of a point from the given surface. This function is the common distance function 
multiplied by minus or plus one depending on which side of the surface a point lies. Since we later apply the level set algorithm only in narrow bands, it is sufficient to calculate the signed distance function only in this narrow band. This method reduces the computational effort of initialization from $O\left(n^{3}\right)$ to $O\left(n^{2}\right)$, where $n$ is the grid resolution in each direction.

\section{EXTENDING THE SPEED FUNCTION AND NARROW BANDING USING A FAST MARCHING METHOD}

In most applications the speed function is not known on the whole simulation domain, but only at the surface. In order to use the level set method it has to be suitably extended from the known values to the whole simulation domain. This can be carried out iteratively by starting from the points nearest to the surface. Mathematical arguments show [12] that the signed distance function can be maintained from one time step to the next by choosing a suitable extension.

The idea leading to fast level set algorithms stems from observing that only the values of the level set function near its zero level set are essential, and thus only the values at the grid points in a narrow band around the zero level set have to be calculated.

Both extending the speed function and narrow banding require the construction of the distance function from the zero level set in the order of increasing distance. But calculating the exact distance function from a surface consisting of a large number of small triangles is computationally expensive and can be only justified for the initialization. An approximation to the distance function can be computed by a special fast marching method [1], [12].

\section{Stability And THE Courant-Friedrichs-Levy (CFL) Condition}

For advancing the level set function we have used a second order space convex finite difference scheme [4]. Consider $\Delta x, \Delta y, \Delta z$, and $\Delta t$ as discretization steps in space and in time, respectively. A necessary condition for the stability of this scheme is the CourantFriedrichs-Levy (CFL) condition which requires that

$$
\Delta t \cdot F_{\max } \leq \min (\Delta x, \Delta y, \Delta z) .
$$

The CFL condition guarantees that the front can cross no more than one grid cell during each time step. In order to have a stable simulator based on the finite difference method, the CFL condition must definitely be satisfied [7].

However, there is a problem stemming from the CFL condition, which limits the simulator performance. If we increase the spatial resolution by $\lambda$, assuming that $F_{\text {max }}$ remains constant, we have to reduce the maximum $\Delta t$ by the same factor $\lambda$, which increases the number of simulation steps by $\lambda$ for reaching the same thickness. Furthermore, an increase in spatial resolution by $\lambda$ increases approximately the number of extracted surface elements by $\lambda^{2}$ and then the computational effort of the visibility determination by $\lambda^{4}$. In summary, an increase in spatial resolution by $\lambda$ leads to an increase in simulation time by a factor $\lambda^{5}$, if one uses the most precise visibility determination.

\section{Transport Models}

The transport of the particles above the wafer surface specifies the deposition and etch rate. Assume that within a feature the frequency of particle-particle collisions is negligible relative to particle-surface collisions, that is, we are in the molecular or Knudsen regime [10]. In this case the transport of the particles can be simulated using the radiosity model. In the other case the collision of single particles plays a major role and their concentration is determined by the diffusion equation.

\section{A. Particle Distribution for Deposition and Etching}

For modeling deposition it is assumed that the distribution of the particles coming from the source obeys a cosine function around the normal vector of the plane in which the source lies [10], [11]. This implies that the flux at a surface element is proportional to the cosine of the angle between the connecting line between the center of mass of a surface element and the source and the normal vector of the source plane.

A function which has been used for ions in plasma systems for etching processes is the normal distribution $f(\theta)=(2 \pi \sigma)^{-1 / 2} \cdot \exp \left(-\theta^{2} / 2 \sigma^{2}\right)$ where $\theta$ is the angle around the normal vector of the source plane and the angular width of the distribution is specified by $\sigma$. For the reflections of particles diffuse and specular reflection are assumed for deposition and etching processes, respectively [10].

\section{B. Visibility Determination}

Most of the computation time for simulating the transport of the particles above the wafer by the radiosity model is consumed in determining the visibility between the surface elements which is an $O\left(\mathrm{~m}^{2}\right)$ operation, where $m$ is the number of surface elements growing approximately like $O\left(n^{2}\right)$. If the connecting line between the center of mass of two surface elements does not intersect the surface, i.e., the zero level set, those surface elements are visible from each other. In order to decrease the computational effort related to determining the visibility between the surface triangles, we have assumed that two triangles are visible from each other if the center point of the grid cells in which the triangles are located, are visible from each other. Since there are at least two triangles in each grid cell, considerable time is saved. 


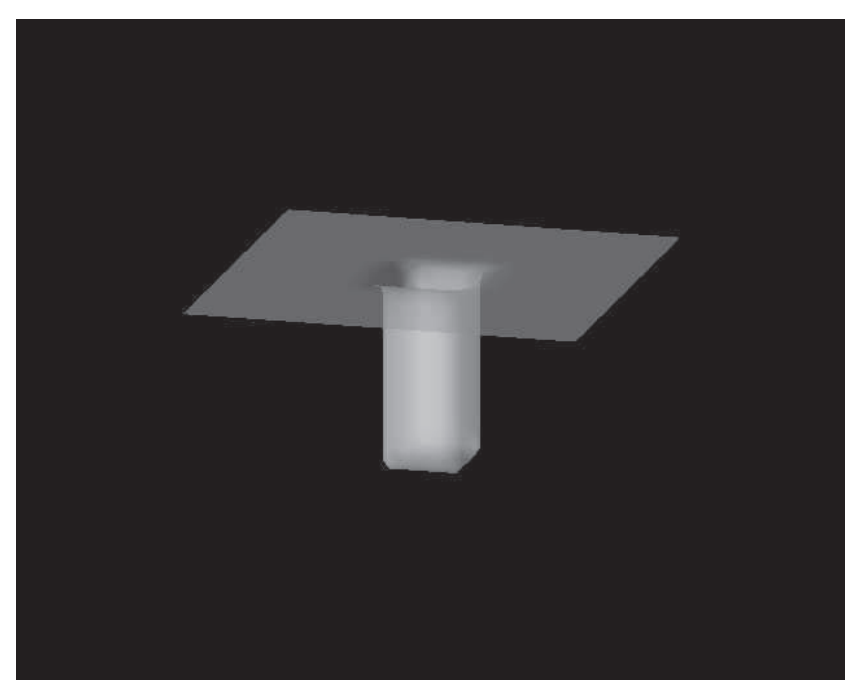

Fig. 1. Initial boundary

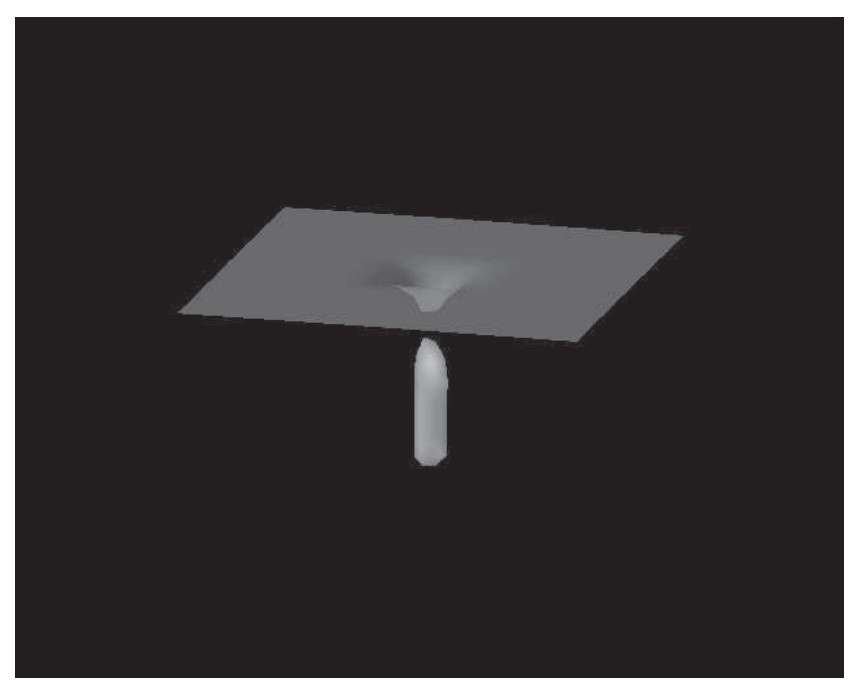

Fig. 2. Simulation result after deposition

\section{Radiosity Model}

The radiosity model assumes that the total flux depends on the flux directly from the source, as well as an additional flux due to the particles which do not stick and are re-emitted. After discretizing the problem the flux vector whose elements are the total flux at different surface elements can be expressed by a matrix eqation. There are two numerical approaches for solving this problem. The first one is to use a direct solver for the matrix equation. Although this is practical in two dimensions [1], it becomes impractical due to the computational effort needed by calculating the inverse matrix for three-dimensional problems. In three dimensions we solve the equation iteratively.

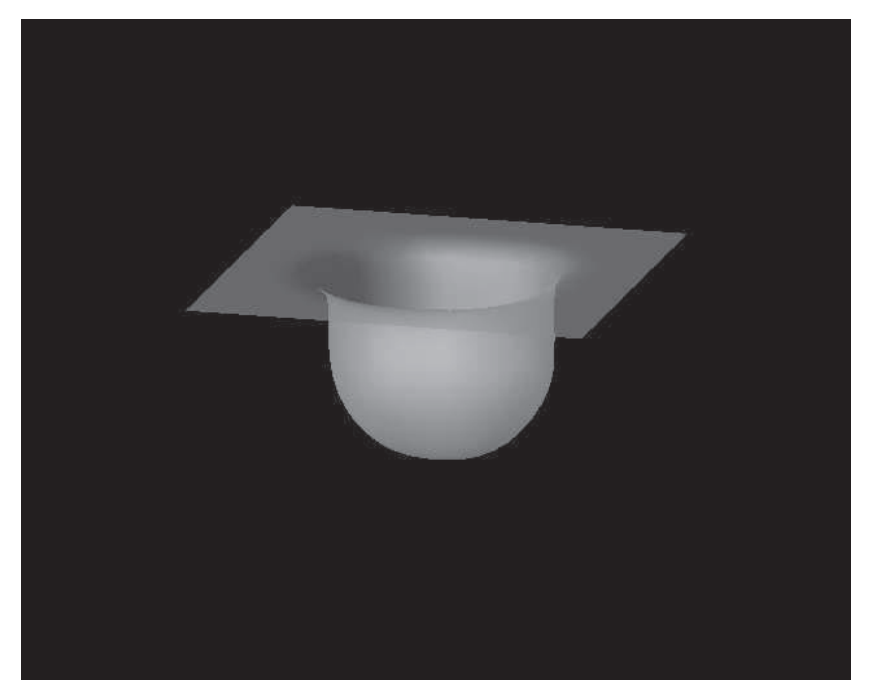

Fig. 3. Simulation result for isotropic etching

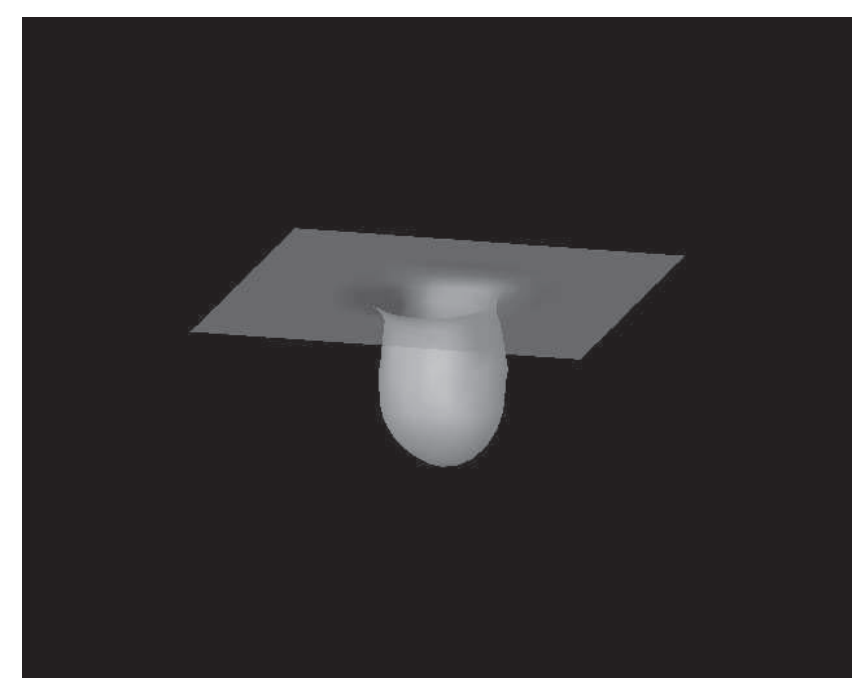

Fig. 4. Simulation result for directional etching

\section{C.1 Iterative Solver}

The iterative solution [12] consists of a series expansion in the interaction matrix. Suitably interpreted, it can be viewed as a multi-bounce model, in which the number of terms in the series expansion corresponds to the number of bounces that a particle can undergo before its effects are negligible. This approach allows to check the error remainder term to determine how many terms must be kept. Since most of the particles either stick or leave the domain after a reasonable number of bounces, this is an efficient approach. By constructing the remainder term, we can measure the convergence of the expansion and keep enough terms to bound the error below a user-specified tolerance. 


\section{Simulation Results}

In this section we present some simulation results for deposition and etching processes. We begin with a source deposition into a rectangular trench shown in Fig. 1. Fig. 2 shows the simulation result of a source deposition from a plane located above the trench leading to void formation including the visibility and shading effects. The particle distribution is a cosine distribution around the normal vector of source plane.

Fig. 3 shows a straightforward simulation of isotropic etching of the same trench from which material is being isotropically etched. As expected, the sides of the trench are cleanly etched away and are rounded.

Finally Fig. 4 shows directional etching of the same trench. The incoming flux at the surface element is a cosine function of the angle between the surface normal and the normal vector of the source plane without visibility effects. The reflection effects are also ignored which is approximately the case for directional etching of ions by a plasma etching process. The trench has been etched less than the selective isotropic etching at the sides and tends to be etched more in vertical direction.

Table I shows a comparison between the simulation times of these different simulation processes for different grid resolutions. The most time consuming simulation is the deposition simulation because all of the time expensive steps, e.g., visibility determination, extending the speed function, and the iterative solver are required. By directional etching, extending the speed function is the only time consuming part of the simulator. Therefore, the simulation time is smaller than for the deposition process. For isotropic etching neither visibility determination, the iterative solver, nor extension the speed function contribute. Thus the simulation time is very small compared to the other simulations. In the third column of Table I the simulation times for a grid resolution two times that of the second column are presented. As an example, for deposition the time has been increased by about a factor of 32 .

TABLE I

Simulation times FOR DIFFERENT SIMULATIONS PRESENTED IN THIS PAPER

\begin{tabular}{|c|c|c|}
\hline Grid resolution & $30 \cdot 30 \cdot 30$ & $60 \cdot 60 \cdot 60$ \\
\hline Deposition time/step & $1.54 \mathrm{~s}$ & $47.4 \mathrm{~s}$ \\
\hline Isotropic etching time/step & $0.53 \mathrm{~s}$ & $2.77 \mathrm{~s}$ \\
\hline Directional etching time/step & $0.97 \mathrm{~s}$ & $21.1 \mathrm{~s}$ \\
\hline
\end{tabular}

\section{Conclusion}

State of the art algorithms for surface evolution processes like deposition and etching processes in three dimensions have been implemented. A general simulator called ELSA was developed based on the level set method combining the narrow banding and fast marching method for extending the speed function. The speed of simulation was improved in several steps, e.g., in initialization, visibility determination, and solving the radiosity matrix. A comparison between the simulation time of different simulation processes was presented that shows how time consuming the different parts of the simulator are. Furthermore, the effect of increasing the grid resolution on the simulation time was shown.

\section{ACKNOWLEDGMENT}

The authors acknowledge the support of Cypress Semiconductor Corporation, USA and Toshiba Corporation, Japan for this work.

\section{REFERENCES}

[1] A. Sheikholeslami, C. Heitzinger, H. Puchner, F. Badrieh, and S. Selberherr, 'Simulation of void formation in interconnect lines,' Proc. of SPIE's first International Symposium on Microtechnologies for the New Millennium: VLSI Circuit and Systems, Gran Canaria, Spain, May 2003, pp. 445-452

[2] C. Heitzinger, J. Fugger, O. Häberlen, and S. Selberherr, 'On increasing the accuracy of simulations of deposition and etching processes using radiosity and the level set method,' Proc. of European Solid-State Device Research Conference, Florence, Italy, September 2002, pp. 347-350

[3] W. Pyka, C. Heitzinger, N. Tamaoki, T. Takase, T. Ohmine, and S. Selberherr, 'Monitoring arsenic in-situ doping with advanced models for poly-silicon CVD,' Proc. of Simulation of semiconductor Processes and Devices, Athens, Greece, September 2001, pp. 124-127

[4] C. Heitzinger, Simulation and Inverse Modeling of Semiconductor Manufacturing Processes, Dissertation, Technische Universität Wien, 2002

[5] W. Pyka, Feature Scale Modeling for Etching and Deposition Processes in Semiconductor Manufacturing, Dissertation, Technische Universität Wien, 2000

[6] U.H. Kwon and W.J. Lee, 'Three-dimensional deposition topography simulation based on new combinations of flux distribution and surface representation algorithms,' Thin Solid Film, vol. 445, pp. 80-89, 2003

[7] O. Runborg, 'Finite difference methods for the advection equation,' Tillä̈pade numeriska methoder II, May 2003

[8] K.K.H. Toh, A.R. Neureuther, and E.W. Scheckler, 'Algorithms for simulation of Three-dimensional Etching,' IEEE Trans. Computer-Aided Design, vol. 13, pp. 616-624, 1994

[9] E.W. Scheckler and A.R. Neureuther, 'Models and algorithms for three-dimensional topography simulation with SAMPLE-3D,' IEEE Trans. Computer-Aided Design, vol. 13, pp. 219-229, 1994

[10] T.S. Cale, 'Flux distributions in low pressure deposition and etch models,' J. Vac. Sci. Technol. B, vol. 9, pp. 2551-2553, 1991

[11] T.S. Cale and G.B. Raupp, 'A unified line-of-sight model of deposition in rectangular trenches,' J. Vac. Sci. Technol. B, vol. 8, pp. 1242-1248, 1990

[12] J. Sethian, Level Set Methods and Fast Marching Methods, Cambridge University Press, Cambridge, 1999 